\title{
Carbon Nanotube Growth from Semiconductor Nanoparticles
}

Daisuke Takagi, ${ }^{\dagger}$ Hiroki Hibino, ${ }^{*}$ Satoru Suzuki, ${ }^{*}$ Yoshihiro Kobayashi ${ }^{\ddagger}{ }^{\ddagger}$ and Yoshikazu Homma ${ }^{\dagger,}, *$

${ }^{\dagger}$ Department of Physics, Tokyo University of Science, Shinjuku, Tokyo 162-8601, Japan

${ }^{\ddagger}$ NTT Basic Research Laboratories, NTT Corporation, Atsugi, Kanagawa 243-0198, Japan

${ }^{\S}$ CREST, Japan Science and Technology Agency, Kawaguchi, Saitama 332-0012, Japan

\section{Supporting Information}
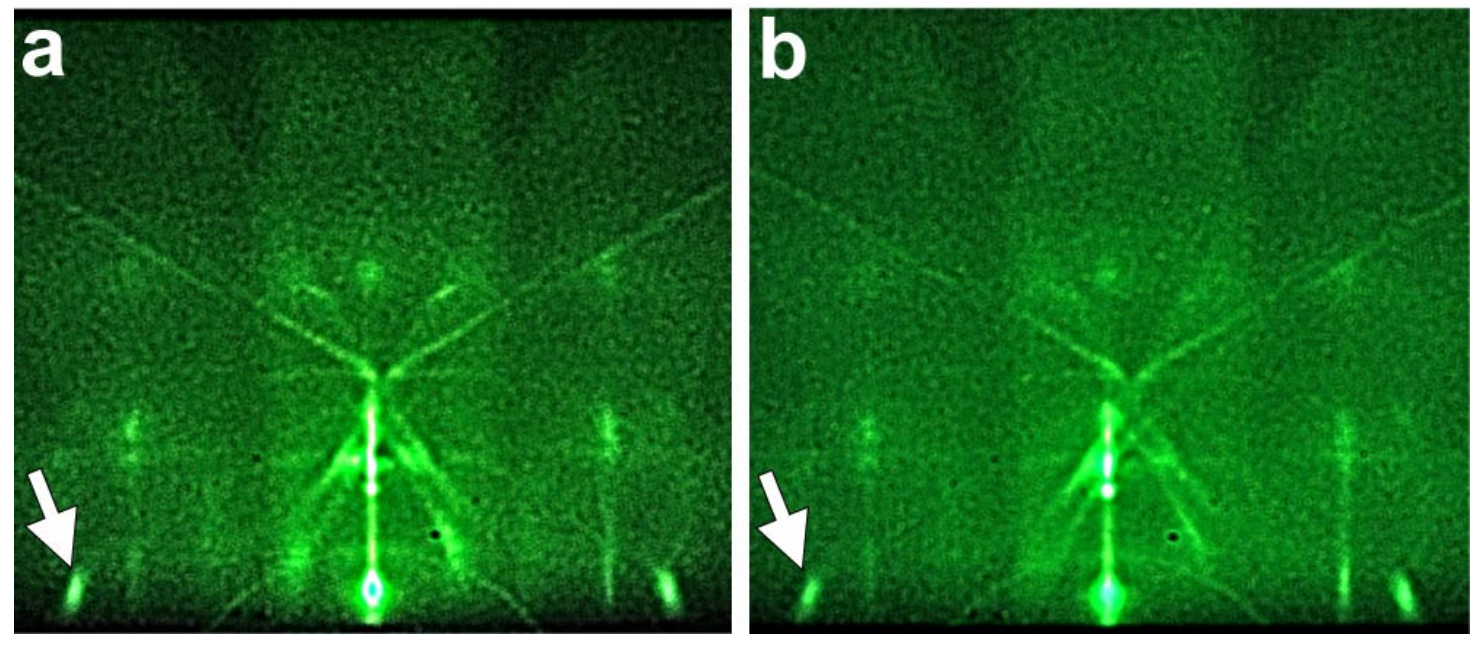

Figure S1. Reflection high energy electron diffraction pattern of epitaxially grown $\mathrm{SiC}$ nanoparticles on $\mathrm{Si}\left(111\right.$ ) observed at (a) $850{ }^{\circ} \mathrm{C}$ and (b) $1000^{\circ} \mathrm{C}$ in ultrahigh vacuum. A $20-\mathrm{kV}$ electron beam was incident from the [112] azimuth. The arrow in each panel indicates a transmission diffraction spot of $\mathrm{SiC}(111)$ plane. 